\title{
Recent advances in the genetic association between osteo- porotic fracture and sarcopenia
}

\author{
Qiaocong Chen ${ }^{\mathrm{a}}$, Huiling Lou ${ }^{\mathrm{a},{ }^{*}}$, Cheng Peng $^{\mathrm{a}}$ \\ ${ }^{a}$ Department of Geriatrics, National Key Clinical Specialty, Guangzhou First People's Hospital, School of Medicine, South \\ China University of Technology, Guangzhou 510180, China.
}

\begin{abstract}
The risk of osteoporotic fracture can be viewed as a function of loading conditions and the ability of the bone to withstand the load. Skeletal loads are dominated by muscle action. Recently, it has become clear that bone and muscle share genetic determinants. Involvement of the musculoskeletal system manifests as bone loss (osteoporosis) and muscle wasting (sarcopenia). There is clinical evidence that osteoporotic fractures are significantly associated with sarcopenia, and sarcopenia may be a potential predictive factor for fracture risk, which suggests that there may be shared genetic determinants between sarcopenia and osteoporotic fracture. In recent years, genome-wide association studies (GWASs) studies have found that both lean mass and hand grip strength are associated with fracture risk, which may provide a possible endophenotype for elucidating the potential genetic study of fracture risk. Our effort to understand the clinical and genetic correlations between osteoporotic fracture and sarcopenia is helpful to understand the interaction between muscle and bone, and to study the etiology of complex musculoskeletal diseases. Identifying potentially important genetic variations in bone and muscle, measuring these variations using state-of-the-art technology, and replicating these experiments in humans and large animals will provide potential drug or intervention targets for osteoporotic fracture valuable in the future.
\end{abstract}

Keywords: Genetics, osteoporosis, fracture, sarcopenia, genome-wide association studies, single nucleotide polymorphism

\section{Introduction}

Osteoporosis is a systemic skeletal disorder characterized by reduced bone mass and strength, and an increased risk of fracture with aging [1]. The definition provided by the World Health Organization (WHO) is a condition where the bone mineral density (BMD) is 2.5 standard deviations (SDs) below the average peak bone mass of healthy females aged 20-29 years, as measured by dual-energy X-ray absorptiometry (DXA) [2]. At present, more than 200 million people worldwide suffer from osteoporosis. Among the population over the age of 50, bone loss leads to a rapid increase in fracture risk and mortality in the elderly, and one in five men or one in three women may suf-

\footnotetext{
* Corresponding author: Huiling Lou, M.D.

Mailing address: Department of Geriatrics, National Key Clinical Specialty, Guangzhou First People's Hospital, School of Medicine, South China University of Technology, Guangzhou 510180, China.

Email: huilinglou@163.com

Received: 16 December 2020 / Accepted: 09 March 2021
}

fer an osteoporotic fracture [3]. The most serious harm of osteoporosis is the occurrence of a fragility fracture, with a predominance in hips, vertebrae, and distal radii, resulting in significant social and economic burdens.

Sarcopenia is defined as the progressive loss of muscle mass and/or muscle function with aging, leading to an increased risk of falls and fractures [4]. In 2018, the European Working Group on Sarcopenia in Older People (EWGSOP) updated the definition of sarcopenia to include three criteria: 1) low muscle strength; 2) low muscle quantity or quality; and 3) low physical performance. Probable sarcopenia is identified by Criterion 1 . The diagnosis is confirmed by additional documentation of Criterion 2. If Criteria 1, 2, and 3 are all met, sarcopenia is considered severe [5]. It is estimated that about 50 million people worldwide suffer from sarcopenia, and the number is expected to reach 500 million by 2050 . The joint occurrence of sarcopenia and osteoporosis was considered an indicator of "dysmobility syndrome", which makes the elderly prone to falls and fractures and has become the main cause of disability and death in the elderly [6]. Among the aging global population, musculoskeletal disorders account for approximately $7.5 \%$ of elderly diseases [7], thus becoming an important public health problem. Prospec- 
tive clinical studies in several countries have shown that patients with hip fractures were complicated with varying degrees of sarcopenia [8], which has proved that sarcopenia is an important risk for osteoporotic fractures.

The risk of osteoporotic fracture can be viewed as a function of loading conditions and the ability of the bone to withstand the load. Skeletal loads are dominated by muscle action. Recently, it has become clear that bone and muscle share genetic determinants. Involvement of the musculoskeletal system manifests as bone loss (osteoporosis) and muscle wasting (sarcopenia). Previous studies have found that bone and muscle tissue are closely related, and both are not only positionally adjacent and biomechanically interrelated, but also connected with tissue metabolism, genetics, endocrine factors, chronic inflammation, malnutrition, exercise, and other systemic factors that collectively regulate skeletal and muscle activity. Patients with sarcopenia and osteoporosis suffer from decreased muscle mass and strength, reduced bone density and mobility, and an increased risk of falls and osteoporotic fractures [9], resulting in decreased quality of life and increased mortality in the elderly.

\section{Clinical evidence for an association between osteoporotic fractures and sarcopenia}

Several clinical studies specifically aimed at the association between osteoporotic fractures and sarcopenia, have been publicated, as discussed below and summarized in Table 1.

Most large cross-sectional studies showed that bone mineral density (BMD) was positively correlated with muscle mass, and decreased muscle mass was an important risk for osteoporotic fractures. A study of 17,891 cases including African Americans, Caucasians, and Chinese, showed that muscle mass and grip strength were positively correlated with $\mathrm{BMD}$, with the risk of bone mass loss/osteoporosis decreased by $37 \%$ for every one standard deviation increase in appendicular lean mass (ALM). Compared to normal individuals, the risk of osteopenia/osteoporosis was 1.8 times higher in patients with sarcopenia [10]. More than 3400 men and women over 60 years old were included in the South Korean Health and Nutrition Survey. Skeletal muscle content, bone mineral density and serum 25 hydroxyvitamin D levels were measured, and the survey showed that sarcopenia combined with vitamin D deficiency group had significantly lower bone mineral density of total hip and femoral neck in both men and women [11]. A study in Shanghai, which enrolled 1766 men and 1778 women aged 18-96 years, showed that the prevalence of sarcopenia was $4.8 \%$ in women and $13.2 \%$ in men over 70 years old, which was close to the prevalence in Japan and South Korea and lower than in Caucasians. Participants' lower limbs and trunk muscle mass were strong predictors of femoral and spine BMD, respectively [12].

Sarcopenia is not only strongly associated with low bone density, but also an important risk for hip fracture. Finnish studies associating sarcopenia and osteoporosis in postmenopausal women divided 590 cases into non-sarcopenic, pre-sarcopenic, sarcopenic, and not classified groups, and showed that sarcopenic women had significantly higher risks of osteoporosis, fracture, and falls during the preceding 12 months compared with non-sarcopenic women, with odds ratios of $12.9,2.7$, and 2.1, respectively [13]. An Italian cross-sectional study of 591 male and female patients admitted to a rehabilitation facility for hip fracture, using the ALMI to diagnose sarcopenia, revealed that $116(21.8 \%)$ females and $52(86.7 \%)$ males had sarcopenia [14]. A Japanese cross-sectional study by Hda et al., which enrolled 2868 male and female patients from health institutions demonstrated 357 had previously suffered hip fractures, and the proportion of patients with sarcopenia and low ALMI in the fracture group was significantly higher than that of the non-fracture group, indicating that aging, low BMD, and sarcopenia are the major risk factors for hip fracture [15].

There are studies that have not found any association between sarcopenia, osteoporosis, and fractures, which may be related to different diagnostic methods of sarcopenia, ethnic and regional differences, the influence of disease status, and medication history [16].

Prospective studies are better able to exclude the interference of multiple confounders and scientifically explore the association of sarcopenia with osteoporosis and fracture. A prospective study enrolled 1089 volunteers with a mean age of 62 years ( $51 \%$ female) with a total of 563 individuals experiencing a fracture during a mean followup of 10.7 years. It was reported that sarcopenic men have lower spine and total body BMD and higher nonvertebral fracture rates and sarcopenic women have lower total hip BMD [17].

A prospective study of 2941 people aged 70 to 79 years with 63 hip fractures occurring during a 6.6 year followup, showed that reduced bone mineral density, muscle mass, muscle strength and function, and increased intermuscular fat mass were associated with an increased risk of hip fracture [18]. A prospective study of about 2000 male community residents of Hong Kong aged over 65, showed that 226 males $(11.3 \%)$ had at least one fracture during the follow-up of 11.3 years. The results showed that sarcopenia was an independent risk factor for fractures besides bone mineral density and other fracture risk factors (hazard ratio $=1.87$ ). If combined with sarcopenia and osteoporosis, the risk of fracture was further increased (hazard ratio $=3.49$ ) [19]. A GERICO cohort study included 913 cases of community residents, with an average age of 65.0 years. The follow-up time was $3.4 \pm 0.9$ years, and 40 cases $(4.4 \%)$ had at least one fragility fracture. The results showed that decreased muscle mass significantly increased fracture risk (odds ratio=2.32). If the sarcopenia combined hip or spine $\mathrm{T}$ score was $<2.5$, the risk of fragility fracture was further increased significantly (odds ratio $=3.39$ ) $[20]$. Therefore, prospective studies have confirmed that sarcopenia and osteoporosis are important risk factors for fragility fracture. Sarcopenia may be con- 
sidered to have a potential predictive value for fracture risk, and joint evaluation of osteoporosis and sarcopenia may help to identify these high-risk fracture patients who need prevention and/or treatment.

A meta-analysis of sarcopenia and falls and fractures in the elderly in 2019 included 17 studies and showed that patients with sarcopenia had a significantly higher risk of fracture than those without sarcopenia (cross-sectional study: combined odds ratio $=1.84$; prospective study: combined odds ratio $=1.71$ ) [8]. The association between sarcopenia and fracture remains significant. The subgroup analysis showed that skeletal muscle loss was significantly associated with fracture independent of study design, population, gender, region, and study quality.

\section{Genetic association between osteoporotic frac- tures and sarcopenia}

Fracture is considered to be the most serious clinical outcome of osteoporosis. Genetic susceptibility, old age, the female gender, falls, and low bone mineral density are the strongest determinants of fracture risk [21]. A positive family history of hip fractures is an important risk factor for osteoporosis and fractures, thus reinforcing the role of genetics in the pathogenesis of osteoporotic fractures [22]. Studies on twins and families with diseases have estimated that the heritability of bone mineral density was as high as $80-90 \%$, while the heritability of lean mass was $70-88 \%$, and the genetic correlation between them was $46 \%$ [23]. The heritability of osteoporotic fractures is $50-60 \%$ [24].
This suggests that genetic factors play an important role in osteoporotic fracture and sarcopenia, but the diseaserelated genetic loci and susceptibility genes found in the present study are far from sufficient to explain the shared genetic susceptibility.

As bone and muscle cells share the same mesenchymal precursor, it is hypothesized that bone and muscle mass share genetic determinants in adults. Since there is clinical evidence that osteoporotic fractures are positively related to sarcopenia, sarcopenia may be a potential predictive factor for fracture risk, suggesting that there may be a sharing of genetic determinants between the development of sarcopenia and osteoporotic fracture.

A meta-analysis of the genetic factors for osteoporosis (GEFOS) and genetic markers for osteoporosis (GENOMOS) consortia [25] (31,016 cases and 102,444 controls) offered a breakthrough in which the strongest relevant BMD markers accounted for about $6 \%$ of BMD variation. In addition, 14 of 56 BMD loci were associated with fracture at a Bonferroni-adjusted significance level, and six of them were reached to a genome-wide significance level ( $\mathrm{p}<5-8)$, including 18p11.21 (FAM210A), 7q21.3 (SLC25A13), 11q13.2 (LRP5), 4q22.1 (MEPE), 2p16.2 (SPTBN1), and 10q21.1 (DKK1). Recent genomewide association studies (GWASs) have confirmed that FAM210A is associated with fracture, BMD, and ALM; then, it was demonstrated that FAM210A is a novel determinant of bone and muscle structure and strength [26], as grip strength, limb lean mass, BMD, and bone strength were reduced in FAM210A knockout mice. Therefore, the FAM210A gene plays a crucial role in regulating bone

Table 1. Published studies for the association between osteoporotic fractures and sarcopenia.

\begin{tabular}{|c|c|c|c|c|c|c|c|c|c|}
\hline Study design & Author & Year & Ethnicity/Country & Gender & Number & $\begin{array}{c}\text { Age (years } \\
\text { mean } \pm \text { SD) }\end{array}$ & Content & $\begin{array}{c}\text { Follow- } \\
\text { up (year) }\end{array}$ & Ref. \\
\hline \multicolumn{10}{|c|}{ Cross-sectional studies } \\
\hline 1 & He et al. & 2016 & $\begin{array}{l}\text { African American, } \\
\text { Caucasian, } \\
\text { and Chinese }\end{array}$ & Male, female & 17,891 & $46.60 \pm 11.8$ & $\begin{array}{l}\text { Sarcopenia, body composi- } \\
\text { tion and osteoporosis }\end{array}$ & & 10 \\
\hline 2 & Lee et al. & 2013 & South Korean & Male, female & 3400 & $>60$ & $\begin{array}{l}\text { Sarcopenia, BMD } \\
\text { and VitD level }\end{array}$ & & 11 \\
\hline 3 & Cheng et al. & 2014 & Chinese & Male, female & 3544 & $18-96$ & Sarcopenia and BMD & & 12 \\
\hline 4 & Sjoblom et al. & 2013 & Finnish & $\begin{array}{l}\text { Postmenopausal } \\
\text { women }\end{array}$ & 590 & $65-72$ & $\begin{array}{l}\text { Sarcopenia and osteoporo- } \\
\text { sis, fracture, fall }\end{array}$ & & 13 \\
\hline 5 & Di Monaco et al. & 2012 & Italian & Male, female & 591 & $80.0 \pm 7.4$ & Sarcopenia and hip fracture & & 14 \\
\hline 6 & Hida et al. & 2013 & Japanese & Male, female & 2868 & & Sarcopenia and hip fracture & & 15 \\
\hline \multicolumn{10}{|c|}{ Prospective studies } \\
\hline 7 & Scott et al. & 2016 & Caucasian & Male, female & 1089 & $62 \pm 7$ & $\begin{array}{l}\text { Sarcopenia, osteoporosis } \\
\text { and nonvertebral fracture }\end{array}$ & 10.7 & 17 \\
\hline 8 & Lang et al. & 2010 & Japanese & Male, female & 2941 & $70-79$ & $\begin{array}{l}\text { Sarcopenia, osteoporosis, } \\
\text { and hip fracture }\end{array}$ & 6.6 & 18 \\
\hline 9 & Yu et al. & 2014 & Chinese & Male, female & 2000 & $>65$ & Sarcopenia and fracture & 11.3 & 19 \\
\hline 10 & Hars et al. & 2016 & Geneva Retirees & Male, female & 913 & $>65$ & Sarcopenia and fracture & $3.4 \pm 0.9$ & 20 \\
\hline Meta-analysis & Yeung et al. & 2019 & $\begin{array}{c}\text { Asian, Australian, } \\
\text { European } \\
\text { and North American }\end{array}$ & Male, female & 52,838 & $\geq 65$ & $\begin{array}{l}\text { Sarcopenia and fracture, } \\
\text { fall }\end{array}$ & & 8 \\
\hline
\end{tabular}


structure and function, which may affect osteoporosis through a biological pathway involving muscle as well as other mechanisms.

Total body-bone mineral density (TB-BMD) measurements are commonly used in children. A GWAS of TBBMD reported that 80 loci associated with TB-BMD explained approximately $10 \%$ of the TB-BMD variance [27]. This publication examined these SNPs in an independent fracture study, where a decrease of one standard deviation in a genetically determined TB-BMD resulted in a $56 \%$ higher probability of fracture. Another phenotype is BMD estimated from a quantitative heel ultrasound, where 12 out of the associated 307 SNPs were associated with fracture risk, and the AQP1 and SLC8A1 loci were newly added as potential genetic determinants of fracture [28].

In the past, most of the genes associated with fracture risk have been identified by detecting known BMD gene loci. To date, there are two GWASs with a vertebral fracture as the endpoint. In the first meta-analysis of GWASs, a single locus on chromosome 16q24 (rs11645938) was associated with the risk of radiographic vertebral fracture, which was not repeated in 5720 cases and 21,791 controls [29]. Another recent meta-analysis of GWASs reported that the chromosomal 2q13 locus was significantly associated with clinical vertebral fractures independent of bone mineral density [30]. The first GWAS of non-vertebral osteoporotic fractures $(n=700)$ was conducted in elderly Chinese and ALDH7A1 was identified as the fracture-related site [31]. However, the gene was not replicated in any large meta-analyses in Europe. In 2018, Trajanoska et al. [32] conducted a GWAS of the largest osteoporotic fractures, involving 37,857 cases and 227,116 controls, which were replicated in nearly 300,000 people (including 147,200 cases). Overall, this effort identified 15 fracture loci with moderate validity (SPTBN1, CTNNB1, ESR1, RSOP3, SHFM1, MBL2/DKK1, LRP5, SOST, CPED1/WNT16, GRB10/COBL, FUPB3, RPS6KA5, STARD3NL, ETS2, and FAM210A loci). Additionally, the RSPO3, ESR1, GRB10/COBL, and ETS2 loci were added to the list as novel fracture loci. Interestingly, all identified loci are known as BMD loci. In general, these SNPs had less effect on fracture than on bone mineral density. Therefore, the genetic effects of all types of fractures are mediated through gene regulation of BMD.

However, the risk of fracture is not entirely determined by the intrinsic nature of bone, as other external factors are known to play a role, such as sarcopenia and fall-related factors. Therefore, it is increasingly recognized that osteoporosis does not account for all low-trauma fractures [22]. Similar to other characteristics and age, the genetic capacity to identify fracture risk also decreases with age. Studying phenotypes associated with fracture risk, such as bone mineral density, lean mass, and grip strength, maybe a good choice to understand the genetic basis of fracture risk [33].

Given that bone and muscle cells come from the same mesenchymal precursor, and that muscle and bone are directly linked, it is thus plausible that there are underlying pleiotropic genes that determine both traits. A high genetic correlation between femoral geometric parameters and total body lean mass was reported in U.S. white adults from Nebraska, ranging from 0.28 to 0.69 [34]. In addition, a recent bivariate genomic linkage analysis found that two chromosomal regions, $5 \mathrm{q} 35$ and $10 \mathrm{q} 24$, had a pleiotropic effect on these phenotypes in the same sample [35]. Our preliminary results in the Framingham Osteoporosis study show a high bivariate genetic correlation between leg lean mass and the cross-sectional geometry of the femur, ranging from 0.56 to 0.81 [36].

At present, studies have reported that AR, ESR, IGF-1, myostatin (GDF8 gene), VDR, LRP5, and IL6 are candidate genes for regulating bone and muscle metabolism through various pathways, including inflammation, growth hormone, and steroid metabolism. Other possible candidate genes, include leptin (LEP), transcription factor SRY box 17 (SRY-17), pleiotropic growth factor (PTIN), resistin (RETN), vascular endothelial growth factor (VEGF), and the glucocorticoid receptor (GCR) [33].

IGF-1 encodes a key enzyme in the steroid hormone pathway producing progesterone, adrenocorticotropic hormone, glucocorticoid, estrogen, and androgen. It acts along the GH/IGF-1 axis and jointly affects the development and growth of bone and muscle [37]. The vitamin $\mathrm{D}$ receptor (VDR) gene has been considered as one of the genetic determinants of bone state, which regulates bone homeostasis through the vitamin D endocrine system. Vitamin D supplementation can increase bone mineral density or bone strength, and also reduce the incidence of falls and fractures. This phenomenon may be due to the relationship between VDR polymorphism and muscle strength [38, 39]. Multiple studies have provided evidence that VDR polymorphism is associated with skeletal geometry [40], fracture [38], and muscle mass or strength [41, 42].

LRP5, a Wnt common receptor, has been proven to be related to BMD and is very important for bone metabolism [43]. Genetic variation studies found that the polymorphism of the LRP5 gene can regulate the relationship between physical activity and bone mineral density in men, suggesting that LRP5 may play a role in bone adaptation to mechanical load in humans [44]. In addition, the Wnt signaling pathway is related to skeletal muscle hypertrophy induced by overload in mice [45]. In conclusion, these studies may indicate that the Wnt signaling pathway, especially LRP5, may be involved in the interaction between bone and muscle (Figure 1). Myostatin is a member of the TGF- $\beta$ superfamily and a negative regulator of skeletal muscle mass [36]. Myostatin may act on bone and muscle tissue upstream of the Wnt signal [46]. IL-6 was proven that high levels of IL- 6 produced by human skeletal muscle after endurance exercise had played a role in mobilization of substrates to support metabolism during exercise [47]. The best study that associated osteoporosis with IL-6 was a SNP G/C mutation at the 174bp, which was shown to be associated with an increased risk of wrist fractures [48], lower hip BMD in postmenopausal 


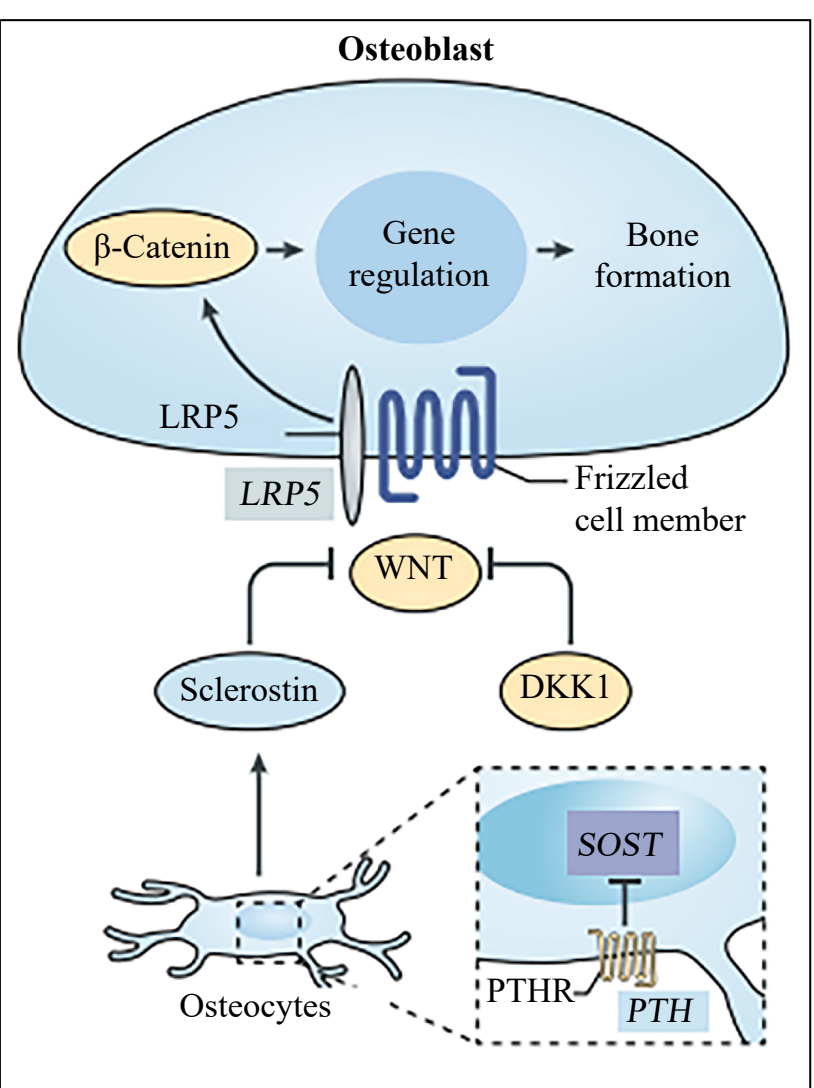

Figure 1. The roles of the Wnt pathway in bone metabolism. LRP5 is a common receptor for WNT pathway signal transduction: the $\beta$-catenin-dependent canonical signal induces bone formation through gene regulation. Also, DKK1 and sclerostin inhibit the Wnt- $\beta$-catenin signaling pathway by binding LRP5.

women [49], and fat-free mass in men but not women [50]. In vitro studies have shown that IL-6 inhibits the secretion of IGF1; therefore, the negative effect of IL-6 on muscle function might be mediated by IGF1 [51].

Bone mineral density and lean mass are hereditary traits. In 2017, Medina Gomez et al. estimated the heritability of shared SNP and performed a bivariate GIS meta-analysis of total body lean mass (TB-LM) and total headless bone mineral density (TBLH-BMD) regions in 10,414 children. The SNP heritability of TBLH-BMD was estimated to be $43 \%$ (95\% CI: $34-52 \%$ ), and that of TB-LM to be $39 \%$ (95\% CI: 30-48\%), and the common genetic component was 43\% (95\% CI: 29-56\%). Eight polymorphic loci with a pleiotropic effect were identified, including seven established BMD loci: WNT4, GALNT3, MEPE, CPED1/ WNT16, TNFSF11, RIN3, and PPP6R3/LRP5. On the TOM1L2/SREBF1 locus, the mutant had opposite effects on TB-LM and TB-BMD, and were strongly correlated with TB-LM. In vitro, SREBF1 was found to be expressed in mice, human osteoblasts, and human muscle tissue. This is the first GWAS meta-analysis using BMD and muscle mass as a bivariate to demonstrate the pleiotropic genetic loci on BMD and lean mass [52]. A recent GWAS suggested that the genes encoding myostatin, actin 3 (actinin-3), peroxisome proliferator-activated receptor $\gamma$ coactivator-1 (PGC-1), myocyte enhancer factor-2C (MEF-
2C), GLYAT, and METTL21C are also closely related to sarcopenia and osteoporosis [53].

In recent years, GWASs have found that both lean mass and hand grip strength are associated with fracture risk [54], which may provide a possible endophenotype for elucidating the potential genetic study of fracture risk. It is thought that this relationship may be due to a negative relationship between muscle strength and balance, and the risk of fall. Zillikens et al. [55] found five SNPs near/in HSD17B11, VCAN, ADAMTSL3, IRS1, and FTO were successfully identified for total lean body mass in 10,1767 individuals, and three SNPs near/in VCAN, ADAMTSL3, and IRS1 for appendicular lean body mass in 73,420 individuals. Karasik et al. [56] identified a new lean mass locus at TNRC6B.

A GWAS of grip strength reported by Willems et al. confirmed that there were 16 novel loci associated with grip strength, including genes related to the structure and function of skeletal muscle fiber (ACTG1), neuron maintenance and signal transduction (PEX14, TGFA, and SYT1) or monogenic syndromes (PEX14, LRPPRC, and KANSL1) related to psychomotor disorders. In addition, the authors also found evidence that bone mineral density and lean mass and grip strength share a common genetic etiology, and suggest that greater grip strength and lower fracture risk have a causal effect [57]. Similar results were found in a study by Trajanoska et al., which found a potential causal relationship between grip strength and fracture risk, but the multitest significance threshold could not be replicated [32]. In addition, our analyses of multiple correlated musculoskeletal traits (i.e., a multivariate GWAS on fracture risk and ALM) have identified several genes with possible pleiotropic loci on both bone and muscle including LPR5, FTO, and PRRX1 using the cFDR method (unpublished data).

\section{Conclusion}

To study the genetic association between osteoporotic fracture and sarcopenia can improve the accuracy of osteoporotic fracture prediction. These loci with large genetic effects on osteoporotic fracture risk might be more valuable in clinical applications, such as drug target design. We should spare no effort to dissect the clinical applications of known risk loci, which is the ultimate goal of all GWASs. For osteoporosis, GWASs have provided genetic support for five of the eight (63\%) approved antiosteoporosis therapeutics [23]. For example, RANKL is the drug target of denosumab and the genetic association between RANKL and BMD was reported by a GWAS [58]. Denosumab is a monoclonal antibody of RANKL as the inhibition of osteoclast activity can increase BMD and reduce the risk of osteoporotic fractures.

GWASs have identified some SNPs or genes associated with fracture risk. However, these variants are only the tip of the iceberg for describing osteoporotic fracture as a complex genetic disease, and are far from enough to ex- 
plain the complex genetic mechanism and pathogenesis of osteoporotic fracture. In order to get better prevention and treatment of the disease, more advanced methods are still needed to mine more pleiotropic genetic loci. This work will help us to better explore the pathogenesis of osteoporotic fracture.

More knowledge of the clinical and genetic correlation between osteoporotic fracture and sarcopenia would be helpful to understand the interaction between muscle and bone, to provide a rationale for studying the etiology of this complex musculoskeletal disease condition, and to create a drug/intervention strategy in future studies. These types of studies could have a huge therapeutic impact on the ability to reverse sarcopenia in the elderly, prevent muscle loss in astronauts, combat falls and fracturesand translate them into new ways to prevent osteoporotic fracture and sarcopenia. Moreover, this strategy will ultimately help to understand broader concepts, such as frailty. Identification of potentially important genetic variations in bone and muscle, measured with state-of-the-art technology, such as the mentioned cFDR, may be useful to explore the multiple causal loci overlapping between osteoporotic fracture and sarcopenia. The replication of experiments in preclinical and clinical studies will provide valuable potential targets and pharmacogenetic applications in osteoporotic fractures.

\section{Declarations}

Authors' contributions: Made substantial contributions to conception and design of the study and performed data analysis and interpretation: Hui-ling Lou; Performed data acquisition, as well as provided administrative, technical, and material support: Cheng Peng.

Financial support and sponsorship: Hui-Ling Lou and Cheng Peng were supported by Guangzhou Planed Project of Science and Technology, Guangzhou, China [201903010091], Medical Science and Technology Foundation of Guangdong Province (CN), [A2019005].

Conflict of interest: The authors declare that they have no conflict of interest.

\section{References}

1. Kanis J A, McCloskey E V, Johansson H, et al. Scientific Advisory Board of the European Society for Clinical and Economic Aspects of Osteoporosis and Osteoarthritis (ESCEO) and the Committee of Scientific Advisors of the International Osteoporosis Foundation (IOF). European guidance for the diagnosis and management of osteoporosis in postmenopausal women. Osteoporos Int, 2013, 24(1): 23-57.

2. World Health Organization. Assessment of fracture risk and its application to screening for postmenopausal osteoporosis: report of a WHO study group [meeting held in Rome from 22 to 25 June 1992]. World Health Organization, 1994.

3. Åkesson K, Marsh D, Mitchell P J, et al. Capture the fracture: a best practice framework and global campaign to break the fragility fracture cycle. Osteoporosis International, 2013, 24(8): 2135-2152.

4. Chen L K, Liu L K, Woo J, et al. Sarcopenia in Asia: consensus report of the Asian Working Group for Sarcopenia. Journal of the American Medical Directors Association, 2014, 15(2): 95-101.

5. Cruz-Jentoft A J, Baeyens J P, Bauer J M, et al. Sarcopenia: European consensus on definition and diagnosisReport of the European Working Group on Sarcopenia in Older PeopleA. J. Cruz-Gentoft et al. Age and ageing, 2010, 39(4): 412-423.

6. Binkley N, Krueger D, Buehring B. What's in a name revisited: should osteoporosis and sarcopenia be considered components of "dysmobility syndrome?". Osteoporosis international, 2013, 24(12): 2955-2959.

7. Dawson A, Dennison E. Measuring the musculoskeletal aging phenotype. Maturitas, 2016, 93: 13-17.

8. Yeung S S Y, Reijnierse E M, Pham V K, et al. Sarcopenia and its association with falls and fractures in older adults: a systematic review and meta-analysis. Journal of cachexia, sarcopenia and muscle, 2019, 10(3): 485-500.

9. Yoo J I, Kim H, Ha Y C, et al. Osteosarcopenia in patients with hip fracture is related with high mortality. Journal of Korean medical science, 2018, 33(4).

10. He H, Liu Y, Tian Q, et al. Relationship of sarcopenia and body composition with osteoporosis. Osteoporosis international, 2016, 27(2): 473-482.

11. Lee S G, Lee Y, Kim K J, et al. Additive association of vitamin D insufficiency and sarcopenia wi th low femoral bone mineral density in noninstitutionalized elderly population: the Korea National Health and Nutrition Examination Surveys 2009-2010. Osteoporosis International, 2013, 24(11): 2789-2799.

12. Cheng $Q$, Zhu X, Zhang X, et al. A cross-sectional study of loss of muscle mass corresponding to sarcopenia in healthy Chinese men and women: reference values, prevalence, and association with bone mass. Journal of bone and mineral metabolism, 2014, 32(1): 78-88.

13. Sjöblom S, Suuronen J, Rikkonen T, et al. Relationship between postmenopausal osteoporosis and the components of clinical sarcopenia. Maturitas, 2013, 75(2): 175180.

14. Di Monaco M, Castiglioni C, Vallero F, et al. Sarcopenia is more prevalent in men than in women after hip fracture: a cross-sectional study of 591 inpatients. Archives of gerontology and geriatrics, 2012, 55(2): e48-e52.

15. Hida T, Ishiguro $\mathrm{N}$, Shimokata $\mathrm{H}$, et al. High prevalence of sarcopenia and reduced leg muscle mass in Japanese patients immediately after a hip fracture. Geriatrics \& gerontology international, 2013, 13(2): 413-420.

16. Tarantino U, Piccirilli E, Fantini M, et al. Sarcopenia and fragility fractures: molecular and clinical evidence of the bone-muscle interaction. JBJS, 2015, 97(5): 429-437.

17. Scott D, Chandrasekara S D, Laslett L L, et al. Associa- 
tions of sarcopenic obesity and dynapenic obesity with bone mineral density and incident fractures over 5-10 years in community-dwelling older adults. Calcified tissue international, 2016, 99(1): 30-42.

18. Lang T, Cauley J A, Tylavsky F, et al. Computed tomographic measurements of thigh muscle cross-sectional area and attenuation coefficient predict hip fracture: the health, aging, and body composition study. Journal of Bone and Mineral Research, 2010, 25(3): 513-519.

19. Yu R, Leung J, Woo J. Incremental predictive value of sarcopenia for incident fracture in an elderly Chinese cohort: results from the Osteoporotic Fractures in Men (MrOs) Study. Journal of the American Medical Directors Association, 2014, 15(8): 551-558.

20. Hars M, Biver E, Chevalley T, et al. Low lean mass predicts incident fractures independently from FRAX: a prospective cohort study of recent retirees. Journal of bone and mineral research, 2016, 31(11): 2048-2056.

21. Koromani F, Trajanoska K, Rivadeneira F, et al. Recent advances in the genetics of fractures in osteoporosis. Frontiers in endocrinology, 2019, 10: 337.

22. Kanis J A. Diagnosis of osteoporosis and assessment of fracture risk. The Lancet, 2002, 359(9321): 1929-1936.

23. Yang T L, Shen H, Liu A, et al. A road map for understanding molecular and genetic determinants of osteoporosis. Nature Reviews Endocrinology, 2020, 16(2): 91-103.

24. Deng H W, Mahaney M C, Williams J T, et al. Relevance of the genes for bone mass variation to susceptibility to osteoporotic fractures and its implications to gene search for complex human diseases. Genetic Epidemiology: The Official Publication of the International Genetic Epidemiology Society, 2002, 22(1): 12-25.

25. Estrada K, Styrkarsdottir U, Evangelou E, et al. Genomewide meta-analysis identifies 56 bone mineral density loci and reveals 14 loci associated with risk of fracture. Nature genetics, 2012, 44(5): 491-501.

26. Tanaka K, Xue Y, Nguyen-Yamamoto L, et al. FAM210A is a novel determinant of bone and muscle structure and strength. Proceedings of the National Academy of Sciences, 2018, 115(16): E3759-E3768.

27. Medina-Gomez C, Kemp J P, Trajanoska K, et al. Lifecourse genome-wide association study meta-analysis of total body BMD and assessment of age-specific effects. The American Journal of Human Genetics, 2018, 102(1): 88-102.

28. Kemp J P, Morris J A, Medina-Gomez C, et al. Identification of 153 new loci associated with heel bone mineral density and functional involvement of GPC6 in osteoporosis. Nature genetics, 2017, 49(10): 1468.

29. Oei L, Estrada K, Duncan E L, et al. Genome-wide association study for radiographic vertebral fractures: a potential role for the 16q24 BMD locus. Bone, 2014, 59: 20-27.

30. Alonso N, Estrada K, Albagha O M E, et al. Identification of a novel locus on chromosome $2 \mathrm{q} 13$, which predisposes to clinical vertebral fractures independently of bone density. Annals of the rheumatic diseases, 2018, 77(3): 378-385.
31. Guo Y, Tan L J, Lei S F, et al. Genome-wide association study identifies ALDH7A1 as a novel susceptibility gene for osteoporosis. PLoS Genet, 2010, 6(1): e1000806.

32. Trajanoska K, Morris J A, Oei L, et al. Assessment of the genetic and clinical determinants of fracture risk: genome wide association and mendelian randomisation study. bmj, 2018, 362.

33. Karasik D, Kiel D P. Genetics of the musculoskeletal system: a pleiotropic approach. Journal of Bone and Mineral Research, 2008, 23(6): 788-802.

34. Sun X, Lei S F, Deng F Y, et al. Genetic and environmental correlations between bone geometric parameters and body compositions. Calcified tissue international, 2006, 79(1): 43-49.

35. Deng F Y, Xiao P, Lei S F, et al. Bivariate whole genome linkage analysis for femoral neck geometric parameters and total body lean mass. Journal of Bone and Mineral Research, 2007, 22(6): 808-816.

36. Li R, Tsaih S W, Shockley K, et al. Structural model analysis of multiple quantitative traits. PLoS Genet, 2006, 2(7): e114.

37. Szulc P, Delmas P D. Bone width is correlated positively with the upper to the lower segment ratio in elderly men-The MINOS study. Bone, 2007, 40(1): 194-199.

38. Uitterlinden A G, Ralston S H, Brandi M L, et al. The association between common vitamin $\mathrm{D}$ receptor gene variations and osteoporosis: a participant-level metaanalysis. Annals of internal medicine, 2006, 145(4): 255264.

39. Arai H, Miyamoto K I, Yoshida M, et al. The polymorphism in the caudal-related homeodomain protein Cdx-2 binding element in the human vitamin $\mathrm{D}$ receptor gene. Journal of Bone and Mineral Research, 2001, 16(7): 1256-1264.

40. Fang Y, Van Meurs J B J, Rivadeneira F, et al. Vitamin D receptor gene haplotype is associated with body height and bone size. The Journal of Clinical Endocrinology \& Metabolism, 2007, 92(4): 1491-1501.

41. Windelinckx A, De Mars G, Beunen G, et al. Polymorphisms in the vitamin $D$ receptor gene are associated with muscle strength in men and women. Osteoporosis International, 2007, 18(9): 1235-1242.

42. Corbetta S, Englaro P, Giambona S, et al. Lack of effects of circulating thyroid hormone levels on serum leptin concentrations. European Journal of Endocrinology, 1997, 137(6): 659-663.

43. Cnossen W R, te Morsche R H M, Hoischen A, et al. Whole-exome sequencing reveals LRP5 mutations and canonical Wnt signaling associated with hepatic cystogenesis. Proceedings of the National Academy of Sciences, 2014, 111(14): 5343-5348.

44. Kiel D P, Ferrari S L, Cupples L A, et al. Genetic variation at the low-density lipoprotein receptor-related protein 5 (LRP5) locus modulates Wnt signaling and the relationship of physical activity with bone mineral density in men. Bone, 2007, 40(3): 587-596.

45. Armstrong D D, Esser K A. Wnt/ $\beta$-catenin signaling activates growth-control genes during overload-induced 
skeletal muscle hypertrophy. American Journal of Physiology-Cell Physiology, 2005, 289(4): C853-C859.

46. Steelman CA, Recknor JC, Nettleton D, Reecy JM. Transcriptional profiling of myostatin-knockout mice implicates Wnt signaling in postnatal skeletal muscle growth and hypertrophy. FASEB J. 2006;20(3):580-2.

47. Solomon A M. Bouloux PM. Modifying muscle mass-the endocrine perspective. J Endocrinol, 2006, 191: 349360.

48. Nordström A, Gerdhem P, Brändström H, et al. Interleukin-6 promoter polymorphism is associated with bone quality assessed by calcaneus ultrasound and previous fractures in a cohort of 75-year-old women. Osteoporosis international, 2004, 15(10): 820-826.

49. Ferrari S L, Karasik D, Liu J, et al. Interactions of interleukin-6 promoter polymorphisms with dietary and lifestyle factors and their association with bone mass in men and women from the Framingham Osteoporosis Study. Journal of Bone and Mineral Research, 2004, 19(4): 552-559.

50. Roth S M, Schrager M A, Lee M R, et al. Interleukin-6 (IL6) genotype is associated with fat-free mass in men but not women. The Journals of Gerontology Series A: Biological Sciences and Medical Sciences, 2003, 58(12): B1085B1088.

51. Barbieri M, Ferrucci L, Ragno E, et al. Chronic inflammation and the effect of IGF-I on muscle strength and power in older persons. American Journal of PhysiologyEndocrinology and Metabolism, 2003, 284(3): E481E487.

52. Medina-Gomez C, Kemp J P, Dimou N L, et al. Bivariate genome-wide association meta-analysis of pediatric musculoskeletal traits reveals pleiotropic effects at the SREBF1/TOM1L2 locus. Nature communications, 2017, 8(1): 1-11.

53. Urano $\mathrm{T}$, Inoue $\mathrm{S}$. Recent genetic discoveries in osteoporosis, sarcopenia and obesity. Endocrine journal, 2015: EJ15-0154.

54. Harvey N C, Kanis J A, Liu E, et al. Appendicular lean mass and fracture risk assessment: implications for FRAX® and sarcopenia. 2019.

55. Zillikens M C, Demissie S, Hsu Y H, et al. Large metaanalysis of genome-wide association studies identifies five loci for lean body mass. Nature communications, 2017, 8(1): 1-13.

56. Weedon M N. Disentangling the genetics of lean mass. 2019.

57. Willems S M, Wright D J, Day F R, et al. Large-scale GWAS identifies multiple loci for hand grip strength providing biological insights into muscular fitness. Nature communications, 2017, 8(1): 1-12.

58. Sabik O L, Farber C R. Using GWAS to identify novel therapeutic targets for osteoporosis. Translational Research, 2017, 181: 15-26.

Cite this article as: Chen Q, Lou H, Peng C. Recent advances in the genetic association between osteoporotic fracture and sarcopenia[J]. Aging Pathobiology and Therapeutics, 2021, 3(1): 02-09. 